\title{
Pseudotumoural soft tissue lesions of the hand and wrist: a pictorial review
}

\author{
Filip M. Vanhoenacker • Michiel Eyselbergs • \\ Erik Van Hul • Pieter Van Dyck • \\ Arthur M. De Schepper
}

Received: 29 October 2010 /Revised: 27 November 2010/Accepted: 27 January 2011 / Published online: 25 February 2011

(C) European Society of Radiology 2011

\begin{abstract}
Mimickers of soft tissue tumours in the hand and wrist are more frequent than true neoplastic lesions. Pseudotumours belong to a large and heterogeneous group of disorders, varying from normal anatomical variants, cystic lesions, post-traumatic lesions, skin lesions, inflammatory and infectious lesions, non-neoplastic vascular lesions, metabolic disorders (crystal deposition disease and amyloidosis) and miscellaneous disorders. Although the imaging approach to pseudotumoural lesions is often very similar to the approach to "true" soft tissue tumoral counterparts, further management of these lesions is different. Biopsy should be performed only in doubtful cases, when the diagnosis is unclear. Therefore, the radiologist plays a pivotal role in the diagnosis of these lesions. Awareness of the normal anatomy and existence and common imaging presentation of these diseases, in combination with relevant clinical findings (clinical history, age, location and skin changes), enables the radiologist to make the correct diagnosis in most cases, thereby limiting the need for invasive procedures.
\end{abstract}

Keywords Pseudotumours · Hand · Wrist · MRI ·

Ultrasound

F. M. Vanhoenacker $(\bowtie) \cdot$ M. Eyselbergs

Department of Radiology,

AZ Sint-Maarten, Duffel-Mechelen, Rooienberg, 25,

B-2570 Duffel, Belgium

e-mail: filip.vanhoenacker@telenet.be

F. M. Vanhoenacker · M. Eyselbergs • E. Van Hul • P. Van Dyck •

A. M. De Schepper

Department of Radiology, Antwerp University Hospital UZA,

University of Antwerp UA,

Wilrijkstraat, 10,

2650 Edegem, Belgium

\section{Introduction}

Mimickers of the soft tissue tumours of the hand and wrist belong to a large and heterogeneous group of disorders, varying from normal anatomical variants to ganglion cysts and other pitfalls such as inflammatory and infectious lesions, post-traumatic lesions, skin lesions, non-neoplastic vascular lesions, metabolic disorders (crystal deposition disease and amyloidosis) and miscellaneous disorders.

The purpose of this pictorial review is to propose a useful classification system for pseudotumours of the hand and the wrist, and to describe the imaging features of these disorders. As many of these lesions are reactive or selflimiting, it is important for the radiologist to recognise these lesions as further investigation or significant intervention is often not required.

Normal variants

Anomalous and accessory muscles

Accessory muscles are congenital anatomical variants that may clinically simulate lesions with mass effect. The most frequently encountered accessory muscles of the hand and wrist are the accessory palmaris longus muscle, the extensor digitorum brevis muscle, the extensor indicis proprius, the accessory abductor pollicis minimi muscle and the anomalies of the flexor digitorum muscles [1-3]. An accessory muscle may mimic a leiomyoma $[2,3]$.

The lesion may present as a wrist mass. The palmaris longus and accessory flexor digitorum superficialis muscle may be associated with median or ulnar nerve entrapment.

Ultrasound shows a hypoechoic structure with a typical striated appearance of muscle. Dynamic ultrasound will show changes in the shape of the mass with active muscle 
contraction. Accessory muscles are isointense to muscles on all MR pulse sequences.

\section{Anomalous osseous structures}

Accessory osseous structures such as a carpal boss may sometimes simulate soft tissue masses clinically. A carpal boss consists of a firm osseous prominence on the dorsum of the hand at the base of the second or third metacarpal and adjacent to the capitate and trapezoid bone (Fig. 1). On MRI, there may be reactive bone marrow oedema and associated joint effusion at the carpo-metacarpal joint [4]. Frictional bursitis may occur adjacent to carpal boss [5].

Synovial cyst, ganglion cyst and tendon sheath cyst

Cystic lesions are the most common lesions with mass effect arising in the hand and wrist, accounting for $50-70 \%$ of all soft tissue tumours of the hand and wrist [6]. The terminology of cystic lesions is very confusing. The terms synovial cyst and ganglion cyst are often used interchangeably.

Thirty percent of the lesions are associated with underlying interosseous ligament injury [7]. The elevated intra-articular pressure, due to accumulation of joint fluid in these diseases, causes herniation of joint fluid and synovium through a gap within the joint capsule. A degenerative process associated with mucin production within or adjacent to the joint capsule has been incriminated as another aetiological factor [8].

A tendon sheath cyst consists of a special ganglion cyst subtype located on the course of a tendon sheath. Tendon sheath cysts have been classically described as arising from the first or second annular pulley (A1-A2) of the digital flexor tendon sheath [9] or from the visceral layer of the tendon sheath $[10,11]$. The aetiology of tendon sheath ganglia is still unclear. Previous trauma and localised rupture within the tendon sheath have been suggested, but have never been proven [9]. Tendon sheath cysts should be distinguished from the more rare intratendinous cysts, which are believed to result from recurrent injury to the tendon with subsequent cystic degeneration [10]. Intratendinous ganglia are clinically relevant because they weaken the structure of tendons and may predispose them to rupture [11].

Cystic lesions of the hand and wrist occur most frequently between the second and fourth decades, but may also arise in the paediatric population and the elderly [6]. There is a female predominance [6]. Half of the patients are asymptomatic [7], whereas the others may suffer from chronic wrist pain, tenderness or functional impairment. The lesion may fluctuate in size, particularly during flexion of the wrist.

Most ganglion cysts are located at the dorsal aspect of the wrist (Fig. 2) adjacent to the scapholunate ligament (60-70\%), whereas volar wrist cysts (Figs. 3 and 4) account for approximately 18-20\%. Approximately two thirds of anterior wrist ganglions arise from the radiocarpal joint and one third arise from the scaphotrapezial joint [12]. Volar cysts may cause median and ulnar nerve palsies [13, 14]. Some cysts may adhere directly to the tendon sheath (Fig. 5) or may be located within the tendon [6, 10]. Rarely, cysts arise from the dorsal aspect of the interphalangeal joints, often secondary to Heberden osteoarthritis in elderly patients (Fig. 6) [6, 10].

The role of imaging is to define the cystic nature of those lesions and to demonstrate a possible communication with the joint. This is important for the surgeon, because the
Fig. 1 Carpal boss. a Plain radiograph (lateral view) showing a bony prominence at the dorsal aspect of the carpometacarpal joint (arrow). b Sagittal fat-suppressed (FS) TSE T2-weighted image (WI). Note bone marrow oedema and subchondral cyst formation at the carpo-metacarpal joint (arrows)
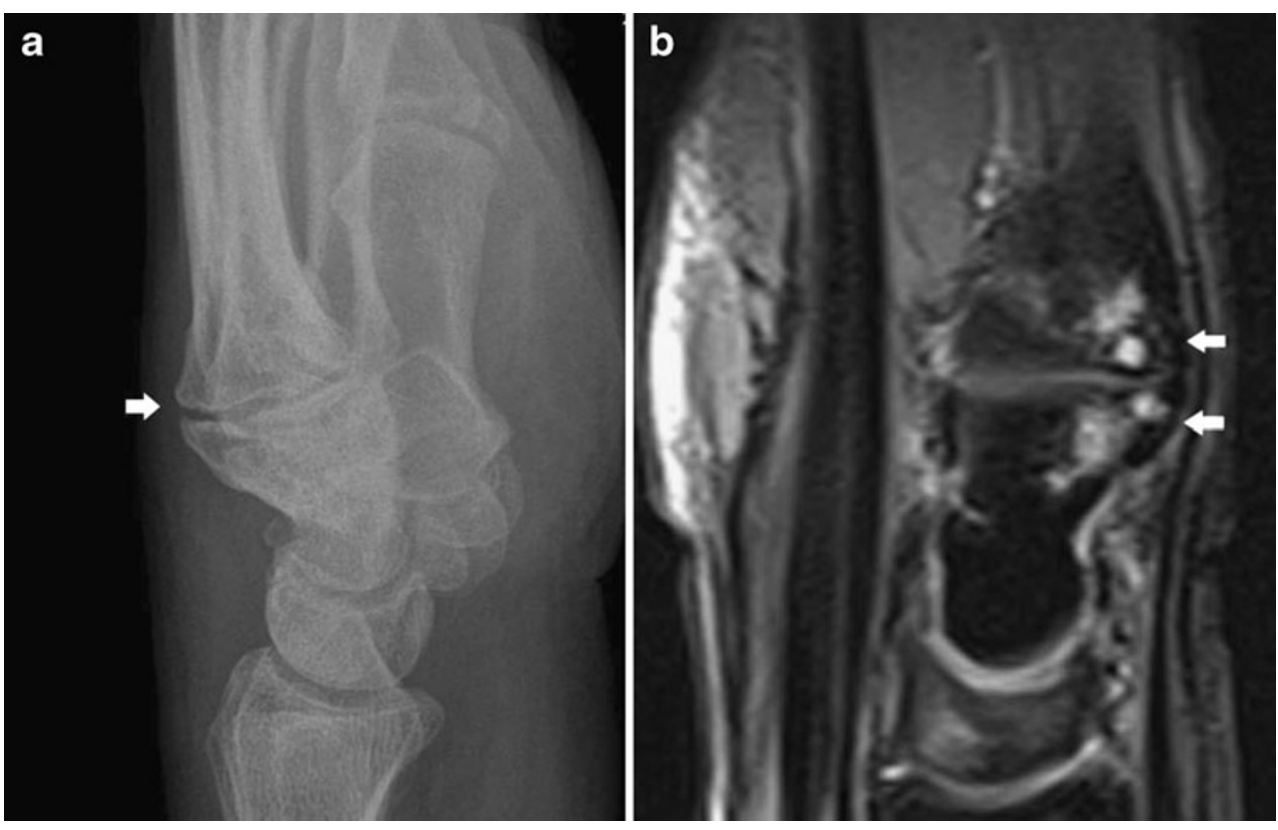

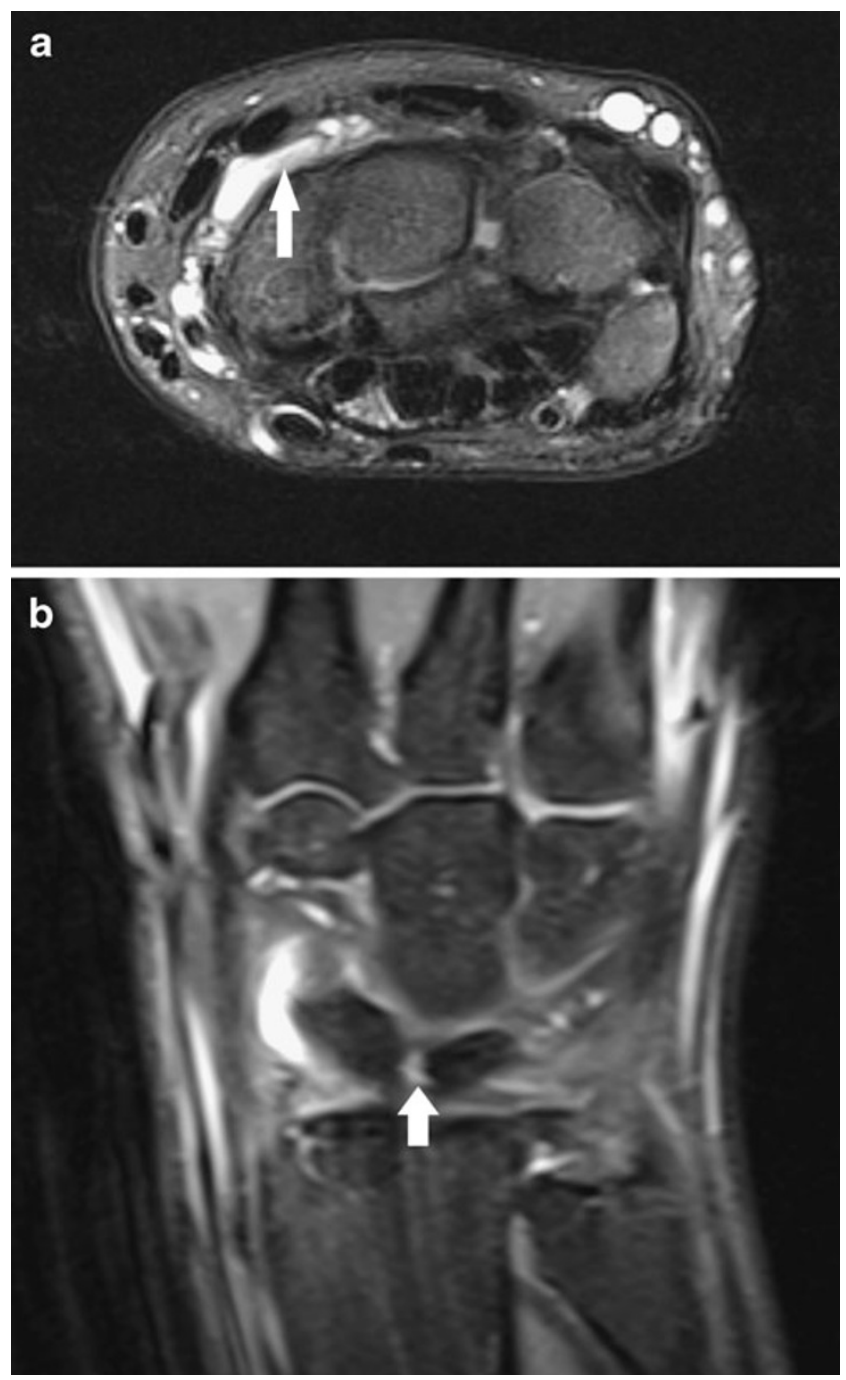

Fig. 2 Small dorsal ganglion cyst. a Axial FS T2-WI. Note a welldelineated hyperintense structure at the dorsal aspect of the wrist (arrow). b The coronal FS TSE T2-WI shows a fluid-filled tear at the scapholunate ligament (arrow)

resection of the communicating stalk with the joint is essential to avoid postsurgical recurrence of the cyst.

Standard radiography is non-specific and may reveal an ill-defined or rounded, non-calcified soft tissue mass. Rarely gas or calcified loose bodies may be seen within a communicating cyst.

Owing to its low soft-tissue contrast, computed tomography (CT) is of limited value in assessing soft-tissue lesions. Cystic lesions are of lower attenuation than muscle and of higher attenuation than fat. Rim enhancement may be seen after intravenous contrast medium administration. Possible communication with the joint is sometimes more difficult to define than on ultrasound and MRI. On ultrasound, synovial cysts and ganglion cysts typically appear as anechoic masses with posterior acoustic enhancement (Figs. 4, 5 and 6), and may have visible communi-
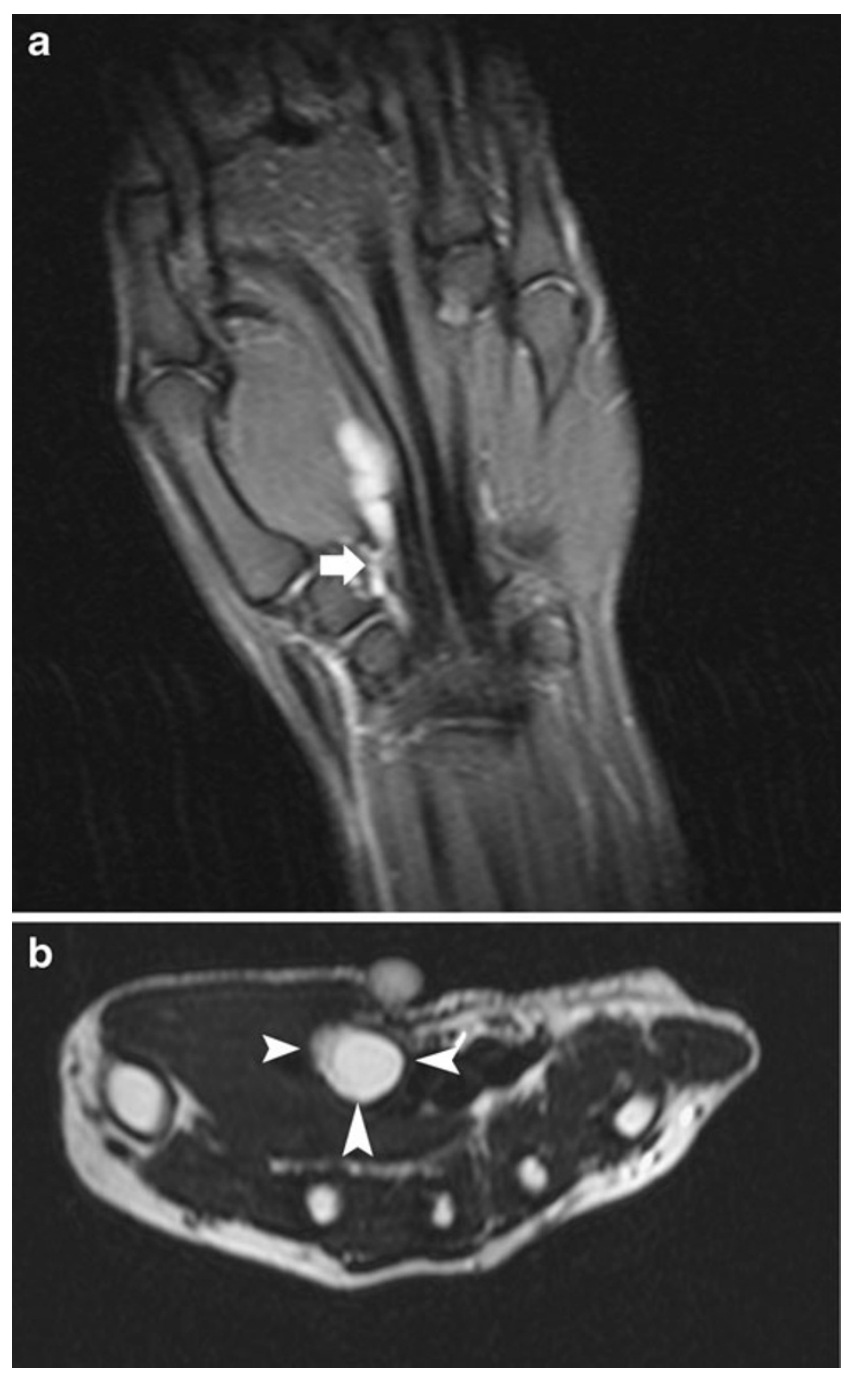

Fig. 3 Volar ganglion cyst. a Coronal FS T2-WI. Note a polylobular hyperintense cystic structure with a small stalk-like connection to the adjacent scaphotrapezial joint (arrow). b Axial FS T2-WI. The cyst is well delineated and is of high signal intensity (arrowheads)

cation with a joint (Fig. 4) or tendon sheath (Fig. 5). Ultrasound is an accurate technique for defining the cystic nature in superficial cysts around the wrist and the hand, but it has limited ability in visualising deeper lying structures and their relationship with the adjacent joint. Dynamic evaluation with ultrasound can differentiate a dorsal ganglion cyst as a well-defined non-compressible anechoic mass from a dorsal radiocarpal joint space recess that compresses under pressure from the ultrasound transducer [15]. The lesion may be multiseptated and may contain some fine internal septations. Volar ganglia are often located on the radial aspect of the wrist, and they may compress and displace the radial artery and the superficial sensory branch of the radial artery [11]. Colour Doppler ultrasound is very useful to depict the relationship between these ventral ganglia and the radial artery. Additionally, 
Fig. 4 Volar ganglion cyst. Longitudinal ultrasound showing a well-defined anechoic structure at the palmar aspect of the carpus. Note a small stalklike connection to the adjacent radiocarpal joint (arrow)

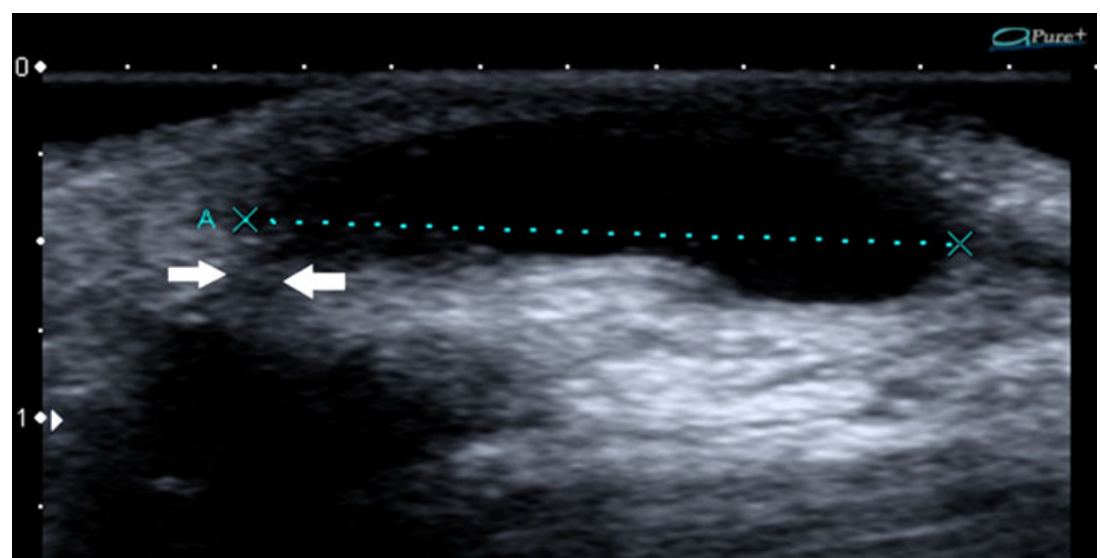

colour Doppler may help to distinguish true cysts from pseudocystic lesions, such as haemangiomas and vascular malformations. The latter lesions usually demonstrate increased Doppler flow, although slow flow in cavernous haemangiomas may not be detected [16]. Although most cystic lesions are completely avascular, a small number may demonstrate peripheral colour Doppler flow due to superimposed inflammation [17]. Ruptured ganglia (due to elevated pressure) have a solid appearance and show increased Doppler signal, both central and peripheral. Diffuse hypoechoic oedema may be seen in the adjacent pericapsular soft tissues. In this scenario, the differential diagnosis with other neoplasms may be difficult if the patient history (acute decrease in size) and lesion location (often located at the dorsal aspect of the wrist) are not taken into account [17]. Furthermore, cysts containing haemorrhagic debris or hyperplastic synovium may simulate solid lesions with mass effect on ultrasound examinations.

If the clinical history (e.g. acute decrease in size suggests a ruptured cyst) and lesion location (e.g. superficial location at the dorsal wrist favours a cyst, deep location leads to the suspicion of another solid soft tissue tumour) are not

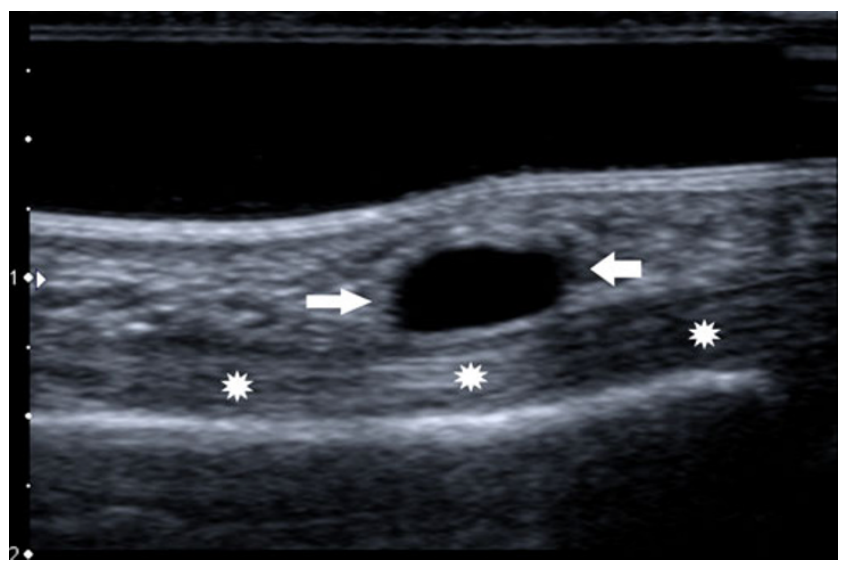

Fig. 5 Cyst of the tendon sheath. Longitudinal ultrasound showing a well-delineated anechoic structure (arrow) on the course of the flexor tendon of the right fourth finger (asterisks) characteristic of a complicated or ruptured cyst, the differential diagnosis should include proliferative synovitis, nodular fasciitis, fibrous tumour, giant cell tumour of the tendon sheath, desmoid tumour and rare malignant soft tissue tumours. MRI with intravenous administration of gadolinium contrast medium is mandatory in these cases [17]. MRI demonstrates the exact location and extent of the cystic lesions, and its relationship to the joint and surrounding structures (Figs. 2 and 3). Cystic lesions are usually well circumscribed, but may be lobulated or multicystic with internal septa. The diagnosis of a typical cyst is usually straightforward by analysis of the signal intensities of the lesion. They are typically hypo- or isointense to muscle on T1-WI, and homogeneously hyperintense on T2-WI. After gadolinium contrast medium administration, subtle rim enhancement of the peripheral fibrovascular tissue in the cyst wall is seen.

However, there are some pitfalls. Atypical cyst content due to debris or haemorrhage may alter the imaging appearance of the cysts. Haemorrhagic cysts may be hyperintense on T1-WI with and without fat suppression because of the presence of methaemoglobin. Gradient echo

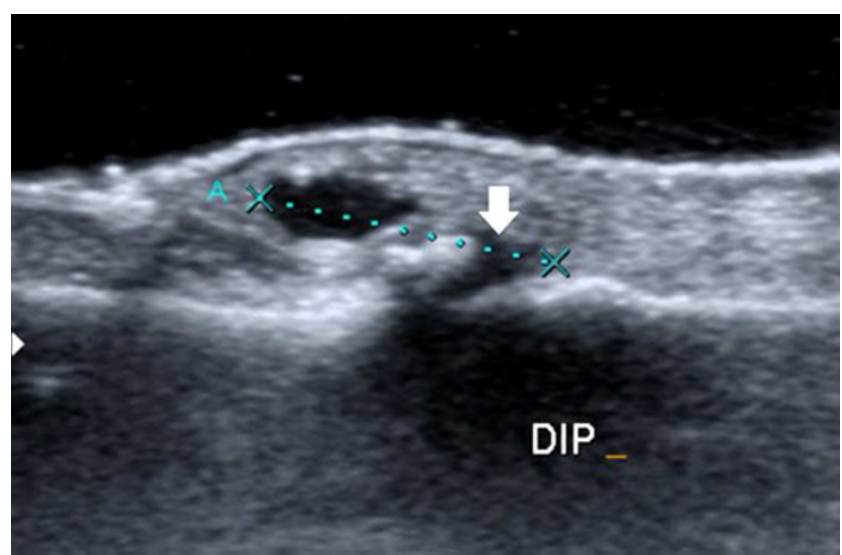

Fig. 6 Small cyst (maximum longitudinal size between calliper measurements) at the dorsal aspect of the distal interphalangeal (DIP) joint in a patient with osteoarthritis. Note a small connecting stalk to the adjacent DIP joint (arrow). Longitudinal ultrasound 
imaging may demonstrate haemosiderin deposition due to previous intracystic bleeding. Whereas haemorrhagic cysts may demonstrate some subtle peripheral enhancement, most solid soft-tissue neoplasms will show more diffuse and central enhancement. Chronic inflammation may cause marked thickening of the synovial membrane and can therefore mimic a solid soft-tissue mass. Ruptured cysts are irregularly delineated and show pericapsular oedema on T2-WI. Although the ruptured cyst itself may show diffuse enhancement after intravenous administration of gadolinium contrast medium, there is absence of enhancement of the pericapsular soft tissue oedema. The presence of non-enhancing peri-capsular oedema is the clue to the differential diagnosis with other solid soft tissue neoplasms.

\section{Post-traumatic lesions}

\section{Myositis ossificans}

Myositis ossificans (MO) is a benign condition of heterotopic bone formation, which can mimic soft-tissue malignancies. It is rarely seen in the hand region [18, 19]. A history of trauma is present in about $60 \%$ of cases. In the acute stage, the most frequent complaint is pain, tenderness and soft tissue swelling.

Imaging findings are time-dependent. In the early/active stage (less than 2-4 weeks), plain films may show a nonspecific soft tissue mass. Faint peripheral calcifications may appear after 7-10 days of presentation (Fig. 7). This subtle peripheral calcification may be more obvious on ultrasound or CT. MRI shows a focal mass that is isointense to slightly hyperintense to muscle on T1-WI. On T2-WI, the lesion is hyperintense (Fig. 7). Contrast enhancement may vary from peripheral rim enhancement to a more diffuse pattern.

In the subacute/intermediate stage (4 weeks to 6 months), a well-defined peripheral calcification and coarser central calcification become apparent on plain radiographs and CT. The ossification pattern is centripetal. MRI shows a peripheral rim of low signal on all pulse sequences, corresponding to calcifications. The signal of the centre of the lesion varies according to the degree of calcification. The peripheral hyperintensity on T2-WI due to perilesional oedema decreases gradually after 4 weeks.

In the chronic or mature stage (after 6 months), the calcification-ossification front further develops following a "zoning" or centripetal pattern, with lamellar bone at the periphery proceeding towards the centre [20-22]. The

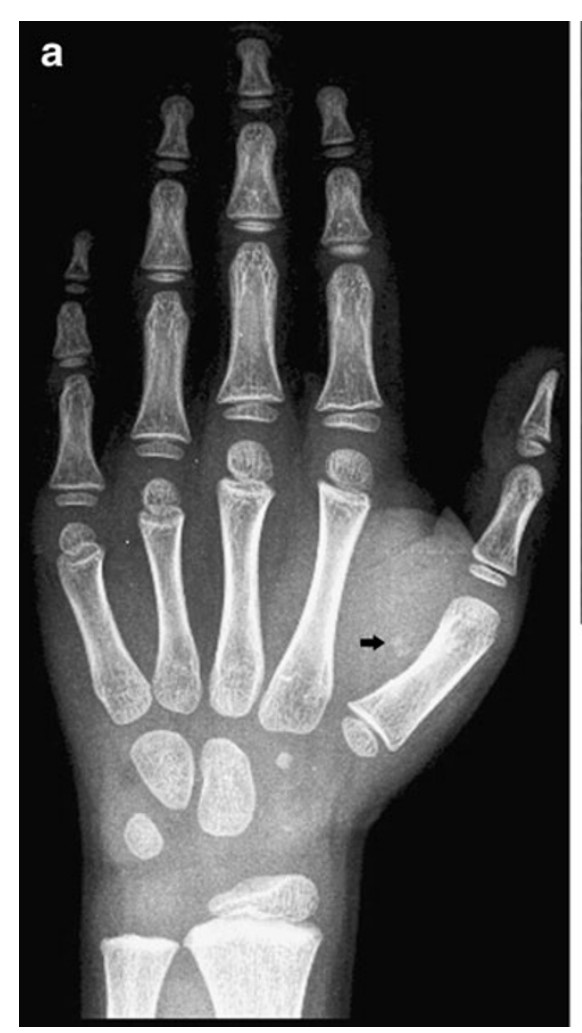

Fig. 7 Myositis ossificans of the thenar in a 7-year-old boy presenting with pain and swelling at the thumb (active phase). a Plain radiograph. Note calcifications (arrow) in the thenar. b Axial SE T2-WI shows an ill-defined intramuscular mass that is hyperintense with central areas

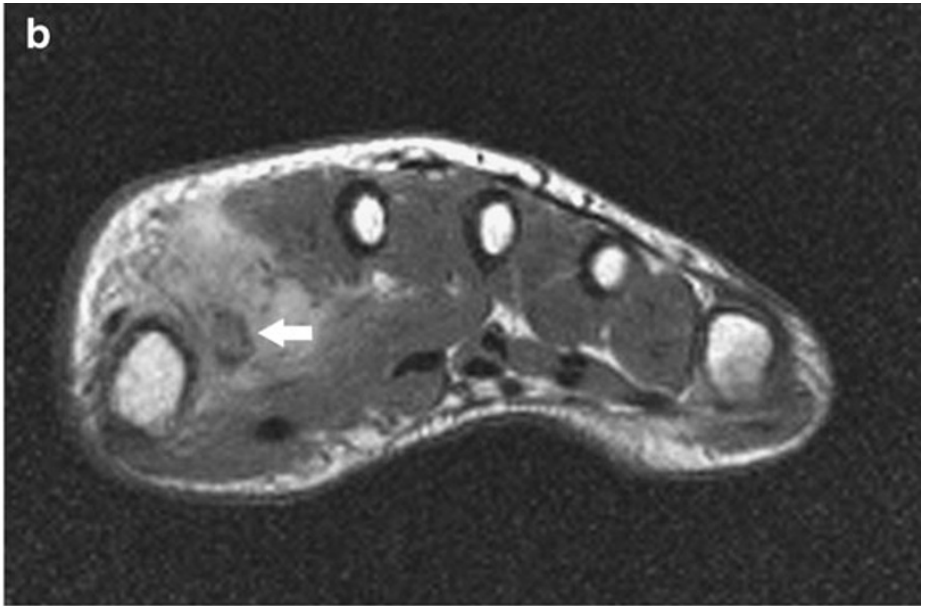

of low signal. Additionally, there is an intralesional area of heterogeneous low signal, corresponding to the calcifications on plain films (arrow) 
lesion is densely calcified or ossified on plain films and CT, and therefore highly reflective on ultrasound with an accompanying retro-acoustic shadowing. On MRI, most lesions are of low signal on all pulse sequences, although areas isointense to normal bone marrow may be seen due to intralesional bone marrow formation. Perilesional oedema is absent on T2-WI [21, 22].

The differential diagnoses for $\mathrm{MO}$ in the acute or subacute stage include muscle abscess, mineralised soft tissue sarcoma (e.g. extraskeletal osteosarcoma and synovial sarcoma), focal myositis and rhabdomyolysis. In contradistinction to calcifying soft tissue sarcoma, myositis has a characteristic peripheral calcification pattern.

The differential diagnoses for mature MO include parosteal osteosarcoma and osteochondroma. In osteochondroma, there is continuity of the medullary and cortical bone of the osteochondroma and the underlying bone. Parosteal osteosarcoma is attached to the underlying bone, whereas mature MO is separated from the underlying bone [21].

\section{Foreign-body granuloma}

Foreign-body granuloma is usually observed after previous penetrating trauma, in which the foreign body is not properly retrieved. Most patients will present with a palpable mass, pain and discomfort.

Plain radiographs are only capable of demonstrating radiopaque foreign bodies, whereas ultrasound is the imaging technique of choice to demonstrate nonradiopaque foreign bodies (Figs. 8 and 9) and the
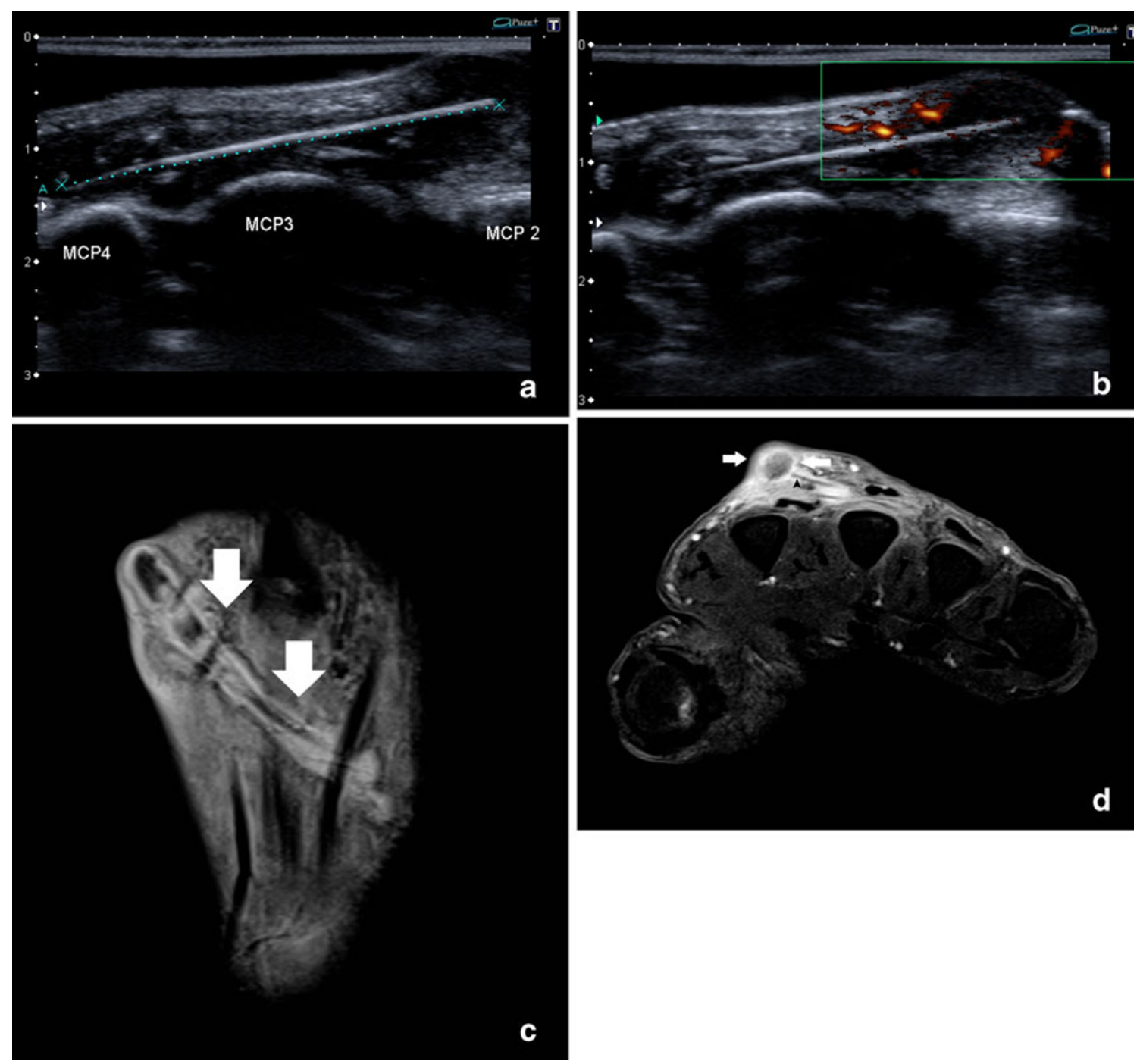

Fig. 8 Foreign body (wood) crossing the metacarpal heads. a Ultrasound shows a central hyperechoic wood fragment surrounded by a hypoechoic inflammatory reaction. b On power Doppler examination, there is increased vascularity at the periphery of the lesion. c Coronal FS T2-WI, showing a hypointense wooden splinter (arrows), with surrounding high signal band, due to inflammatory reaction. d Axial FS T1-WI after intravenous administration of gadolinium contrast medium showing a rim-enhancing abscess (white arrows) adjacent to the wooden splinter (black arrowhead) 


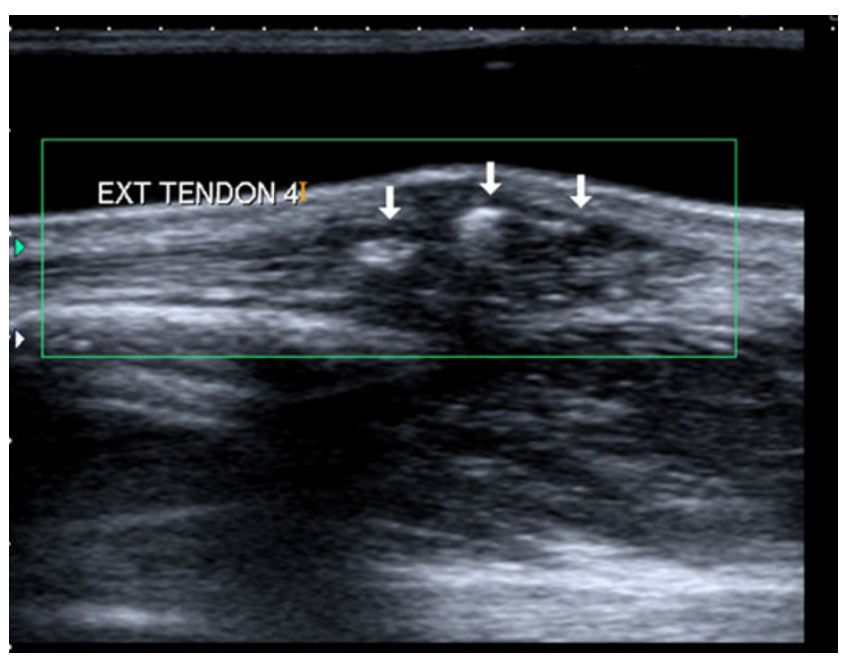

Fig. 9 Foreign body (glass). Longitudinal ultrasound shows multiple hyperechoic reflections with accompanying reverberation artefact (arrows) at the dorsal aspect of the extensor tendons of the fourth digit surrounding inflammatory reaction (Fig. 8). Radiolucent wood or plastic foreign bodies are visualised by ultrasound as brightly echogenic masses that may or may not have posterior acoustic shadowing. An associated inflammatory soft tissue reaction is usually present after $24 \mathrm{~h}$, appearing as a hypoechoic halo surrounding the foreign body with increased vascularity on colour Doppler. The high spatial resolution is important to detect small superficial foreign bodies that can be removed with ultrasound guidance [15].

In the early course of the lesion, high signal on T2-WI and contrast enhancement is seen surrounding the foreign body (Fig. 8), while the granulation tissue may be of low signal on T2-WI without contrast medium uptake in the chronic stage of the disease [20].

\section{Post-traumatic tenosynovitis}

The aetiology of tenosynovitis may be variable, including acute and chronic trauma, infection, inflammatory or metabolic disease (Fig. 10). Post-traumatic tenosynovitis can
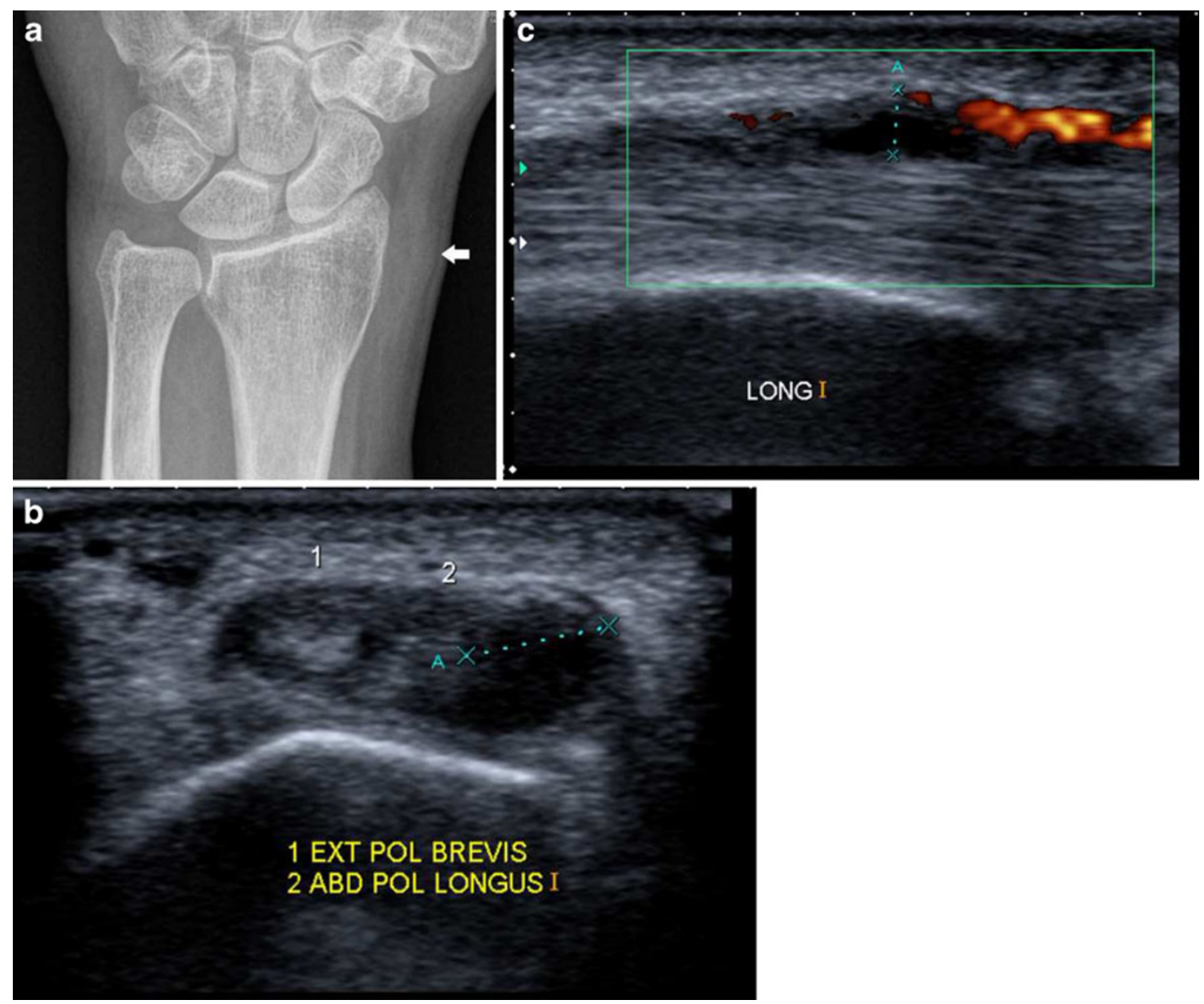

Fig. 10 De Quervain tenosynovitis. a Plain radiograph showing a non-specific soft tissue swelling at the radial styloid process (arrow). b Transverse ultrasound showing fluid surrounding the extensor pollicis brevis and abductor pollicis longus. c Longitudinal power Doppler examination shows increased vascularity surrounding the tendons 
present as a lesion with mass effect if there is sufficient fluid accumulation, synovial hypertrophy or thickening of the retinaculum [5]. On ultrasound, the tendons are surrounded by an anechoic fluid collection. In addition, ultrasound is useful in assessing the internal structure of the tendons, which may be swollen, partially or completely ruptured [5].

Partial tendon tears are difficult to evaluate on ultrasound and appear as focal hypoechoic areas within the tendon. Complete tears will show complete disruption of the normal fibrillar tendon fibres and lack of tendon movement on dynamic ultrasound [16].

Magnetic resonance imaging shows increased hyperintense fluid within the tendon sheath on T2-WI. Although associated calibre and signal intensity changes within the tendons (Fig. 11) may be evaluated on MRI [2], ultrasound is the preferred technique for this purpose.

Cutaneous and subcutaneous lesions

\section{Epidermoid cyst}

Epidermoid cyst formation, also known as epidermal inclusion cyst or infundibular cyst, results from the proliferation of surface epidermal cells within the dermis. The lesion is typically located within the subcutaneous tissue and is often secondary to trauma.

Macroscopically, the lesion is filled with keratin [20]. The cyst wall may rupture with secondary foreign bodytype reaction, granulomatous reaction, granulation tissue or abscess formation [23].

On plain radiographs, epidermal cysts may be located in the subcutaneous tissue or within the bone (most frequently at the terminal phalanges of the fingers). Lesions located within the dermis may cause well-defined osteolysis on the adjacent bone (Fig. 12).

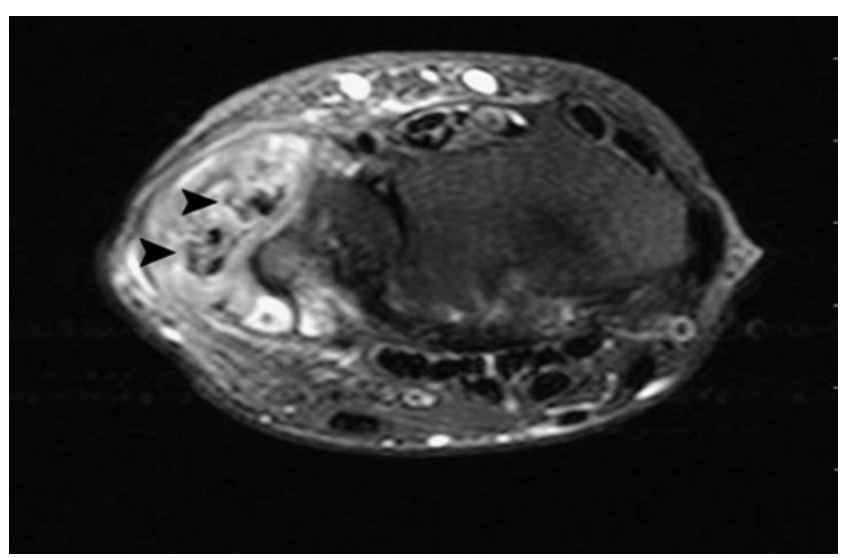

Fig. 11 Extensor carpi ulnaris tenosynovitis. Axial FS T2-WI shows fragmentation into multiple tendon fragments of the extensor carpi ulnaris tendon (arrowheads). Note increased fluid and debris within the tendon sheath
Ultrasound is more sensitive than clinical examination for the diagnosis of an epidermoid cyst. Sensitivity values of 93.5 and $99.3 \%$ have been reported for palpation and ultrasound respectively [24]. The lesion is usually well defined, and echogenicity varies with the content of the cyst.

The lesion is usually hypoechoic, with posterior acoustic enhancement. Intralesional hyperechoic debris or keratin clusters may be seen as well (Fig. 13). Rupture of the cysts may result in changes in shapes (either lobulations, protrusions or abscess pocket formations), pericystic changes and increased vascularity on power Doppler ultrasound [23].

In the case of epidermoid inclusion cysts occurring in the fingertips, there may be bony involvement in which the cyst erodes into the bone causing a lytic lesion within the distal phalanx. Cortical destruction and osteolysis may mimic a malignant or infectious process [6].

On CT and MRI, the lesion is well defined. MRI features depend on the chemical composition of cholesterol and keratin content. Lesions with high lipid content are hyperintense on T1- and T2-WI, whereas the acrystalline form of cholesterol and the presence of keratin and microcalcifications may result in low signal on T2-WI. After administration of intravenous gadolinium contrast medium, there is a lack of contrast enhancement in noncomplicated epidermoid cysts (Fig. 12) [20].

\section{Inflammatory and infectious lesions}

\section{Infectious tenosynovitis and abscess formation}

Although most cases of tenosynovitis are due to overuse trauma, an inflammatory or infectious aetiology may exist as well.

Both ultrasound and MRI are able to distinguish fluid from hypertrophied synovium (pannus), which is impossible on clinical examination alone.

On ultrasound, pannus is displayed as hypoechoic and incompressible synovial thickening compared with mobile and anechoic fluid. Administration of intravenous gadolinium contrast medium can help differentiate pannus from fluid, because pannus demonstrates diffuse enhancement, whereas fluid shows low signal on contrast-enhanced T1-WI [2].

In addition, colour Doppler and contrast-enhanced MRI are useful tools to assess the vascularity of the thickened synovium and disease activity (Fig. 14) [2, 5].

Soft-tissue infection, complicated by abscess formation, may mimic a soft-tissue mass. Infection of the hand and wrist can rapidly spread along the numerous tendon sheaths, fascial planes and lymphatics of the hand and may be accompanied by osteomyelitis. MRI is the preferred 
Fig. 12 Subcutaneous epidermoid cyst with involvement of the adjacent bone of the terminal phalanx of the right fifth finger. a Plain radiograph showing a well-defined osteolytic defect at the radial side of the distal phalanx. Note cortical destruction of the radial cortex. $\mathbf{b}$ Coronal SE T1-WI. The lesion is of intermediate signal intensity (arrows) with some internal areas of relatively high signal. c Coronal TSE T2-WI. High signal intensity of the lesion (arrows). d Coronal FS SE T1-WI after intravenous injection of gadolinium contrast medium. There is only subtle peripheral enhancement (arrows) of the lesion
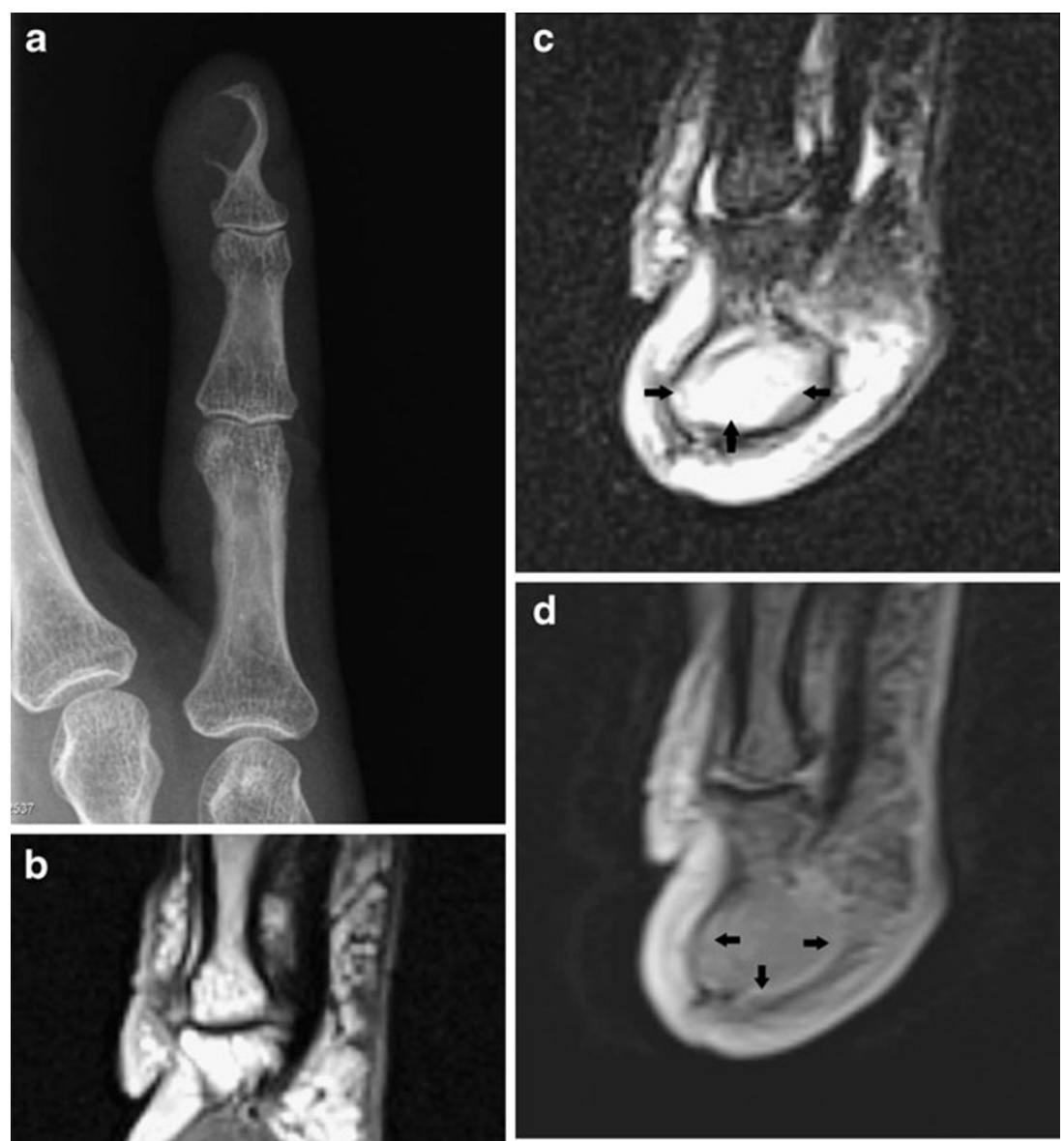

technique to demonstrate the precise extent of the infection. A soft-tissue abscess usually corresponds with low to intermediate signal on T1-WI, high signal on T2-WI and demonstrates peripheral rim enhancement following

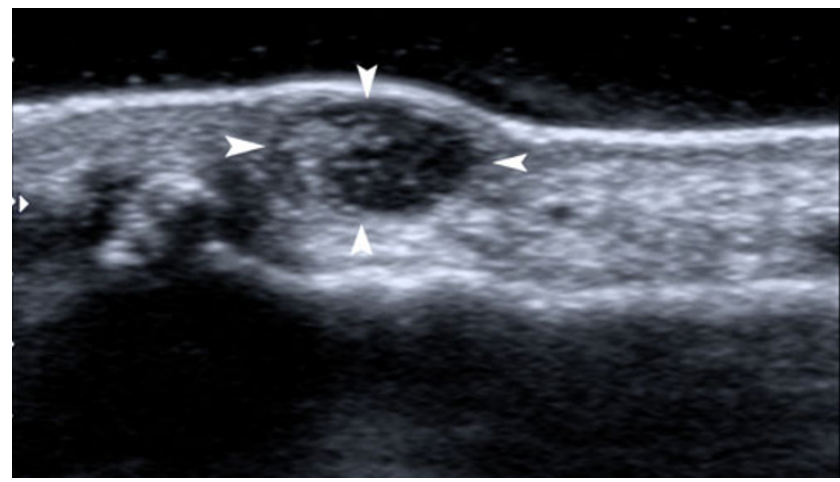

Fig. 13 Subcutaneous epidermoid cyst at the phalanges (arrowheads). Longitudinal ultrasound showing a well-defined subcutaneous lesion with variable echogenicity (anechoic components and some internal hyperechoic debris) intravenous injection of gadolinium contrast medium (Fig. 8) [2]. In the case of associated osteomyelitis, the bone marrow of the adjacent osseous structures will be of high signal on STIR or fat-suppressed T2-WI, and bone marrow enhancement will be seen on contrast-enhanced MRI.

\section{Rheumatoid nodule}

Rheumatoid nodules may be rarely observed in cases of long-standing rheumatoid arthritis or other rheumatic disease such as lupus, spondylarthropathy, agammaglobulinaemia and rheumatic fever. Rarely, it precedes the articular manifestations of rheumatic disease. Histologically, the lesion consists of chronic inflammation, with or without central necrosis.

The lesions are usually located at the dorsal aspect of the hand within the subcutaneous tissue (particularly at sites of pressure or repetitive trauma), but bursae, joints, tendons and ligaments may be involved as well [20]. 

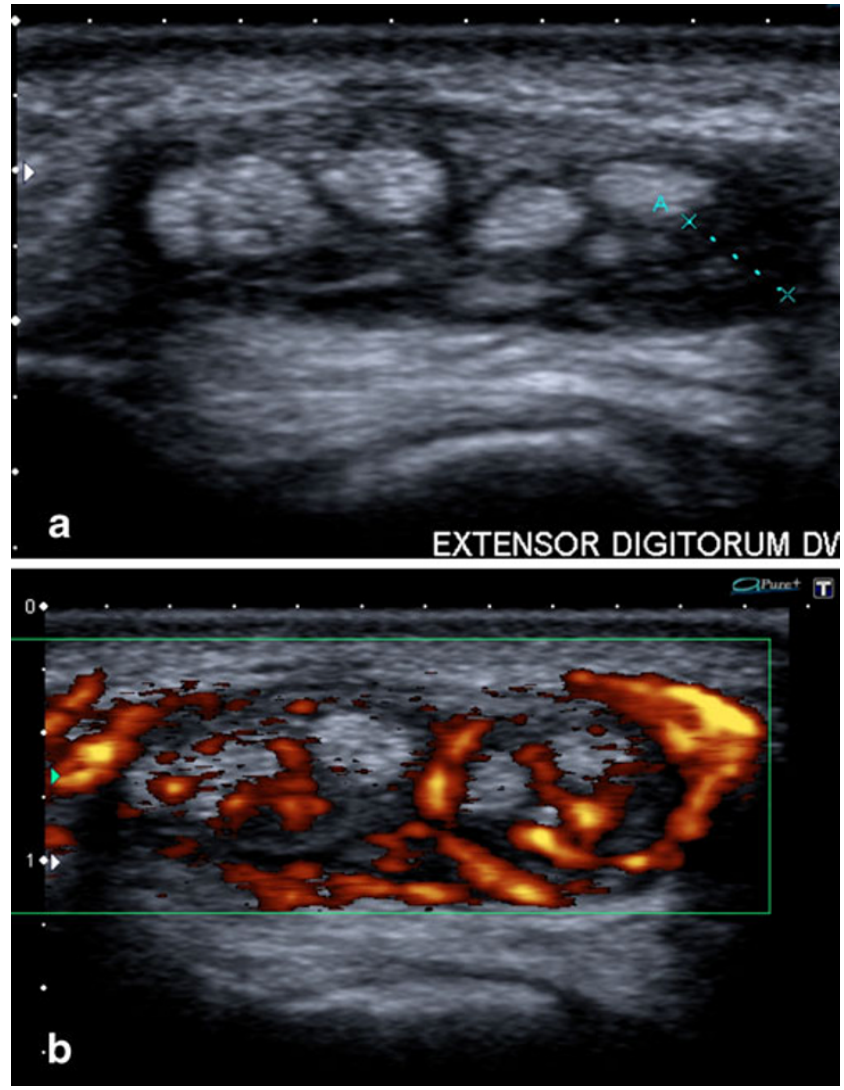

Fig. 14 Inflammatory tenosynovitis in a patient with known rheumatoid arthritis. a Transverse ultrasound of the extensor tendons of the wrist showing fluid surrounding the extensor tendons 4. b Transverse power Doppler examination shows increased vascularity within the tendon sheath and tendons

Ultrasound is non-specific, although the potential value of elastosonography in the differential diagnosis between rheumatoid nodules and gout tophi has recently been reported. Preliminary data suggest that rheumatoid nodules are significantly less elastic than gout tophi [25]. MRI may demonstrate iso- to hypointense lesions compared with muscle on T1-WI. Solid lesions are hypointense on T2-WI, whereas cystic lesions are hyperintense. The enhancement pattern is variable, ranging from marked in solid lesions to peripheral ring-like in cystic lesions (Fig. 15).

\section{Subcutaneous granuloma annulare}

Subcutaneous granuloma annulare (SGA) is a rare inflammatory dermatosis, which may be encountered in children between 2 and 5 years of age. Although the pretibial area and the scalp are the most frequent locations, SGA can manifest anywhere, and the hand may be involved as well $[26,27]$. The lesions are usually solitary but may be multiple. The lesion presents as an ill-demarcated, fastgrowing, painless, subcutaneous lesion. There are no calcifications on imaging. Ultrasound may demonstrate elongated hypoechoic masses with vascularity on colour Doppler [15]. Ultrasound is the preferred imaging technique in young children, because there is no need for anaesthesia. MRI typically shows a subcutaneous mass with ill-defined margins. On T1-WI, the lesion is iso- to slightly hyperintense to muscle. On T2-WI, heterogeneous, predominantly high signal intensity is seen. After administration of gadolinium contrast medium, there is variable (usually diffuse) enhancement [28-35].

Non-neoplastic vascular lesions

\section{Hypothenar hammer syndrome}

A post-traumatic partially thrombosed palmar aneurysm resulting from repeated trauma to the wrist (hypothenar hammer syndrome) may mimic a soft tissue tumour [18].

The typical location of the lesion on the distal part of the ulnar artery distal to the hamulus of the hamate bone, the history of repeated trauma, the signal intensity of the lesion (indicative of thrombosis) and direct demonstration of occlusion of the ulnar artery with different imaging techniques (Doppler ultrasound, MR and CT angiography) may allow a correct diagnosis (Fig. 16) [36-39]. Catheter angiography is rarely required for diagnosis. It may show segmental ulnar artery occlusion in the affected palm or "corkscrew" elongation with alternating stenoses and ectasia.

\section{Metabolic disorders}

Crystal disease such as gout and pseudogout (hydroxyapatite and calcium pyrophosphate dehydrate deposition disease) and amyloid deposition may occasionally present as peri-articular soft-tissue masses at the wrist and fingers (Fig. 17). Clinical and radiographic findings of tophaceous gout or pseudogout are usually diagnostic [40-42]. Calcification is more frequently seen in pseudogout. On ultrasound, tophi appear as heterogeneously hypoechogenic structures with some internal reflections and some shadowing. The presence of adjacent bone erosions is a useful clue to the diagnosis. Increased vascularity can be seen surrounding the tophus [42]. On MRI, tophaceous gout and pseudogout show low to intermediate signal intensity on T1-WI. On T2-WI, the signal varies from heterogeneously hypo- to hyperintense, depending on the degree of inflammation [42]. Diffuse contrast enhancement is the rule [43, 44].

Amyloidosis is a disease complex caused by the extracellular deposition of an insoluble protein called amyloid. Amyloid deposition in and around the joints is usually associated with underlying conditions such as multiple myeloma, chronic infections or inflammation 
Fig. 15 Histopathologically proven rheumatoid nodule in a patient with known rheumatoid arthritis. a Coronal SE T1-WI. Hypointense subcutaneous nodule at the palmar aspect of the distal phalanx of the right digit 3 (arrowheads). b Sagittal FS TSE T2-WI. High signal intensity of the lesion (black arrows). There is moderate pressure erosion of the palmar aspect of the distal phalanx. c Sagittal FS SE T1-WI after intravenous injection of gadolinium contrast medium. There is peripheral enhancement of the lesion (black arrowheads). Palmar localisation of a rheumatoid nodule is less frequent than localisation at the dorsal aspect of the hand
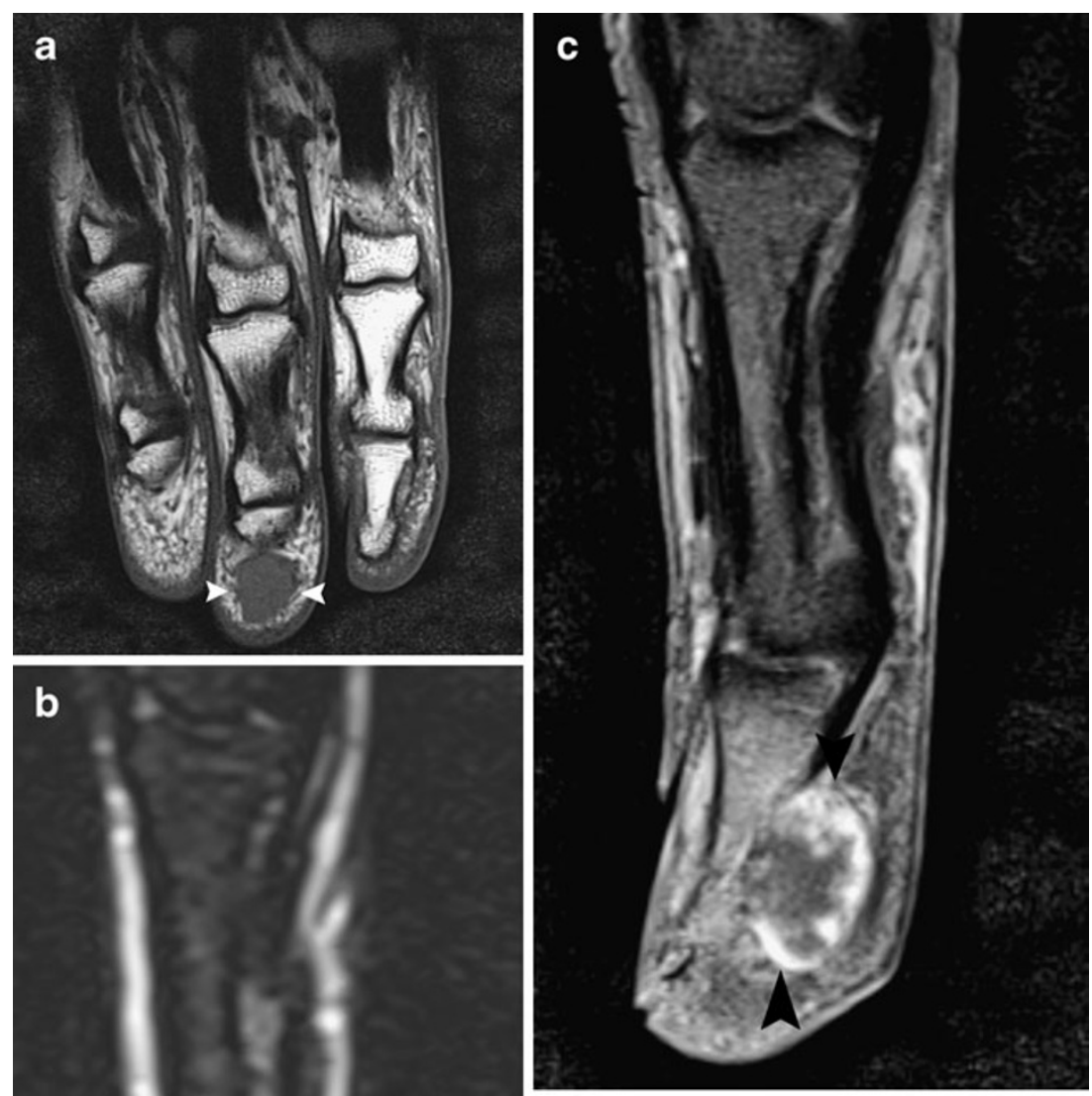

(secondary amyloidosis). Primary musculoskeletal amyloidosis is rare. Any joint may be involved, but the disease most frequently affects the shoulders, hips, wrists and knees. Amyloidosis of the wrist may cause carpal tunnel syndrome.

Radiologically, periarticular soft tissue involvement is usually associated with amyloid arthropathy. Characteristic findings include asymmetric soft tissue masses, absence of periarticular osteoporosis, preservation of the articular space, subchondral cysts, joint effusion and erosions. The preservation of the joint space and absence of juxtaarticular osteoporosis are important clues in the differential diagnosis with rheumatoid arthritis [45]. Soft-tissue amyloid deposition typically shows low to intermediate signal intensity on T1- and T2-WI. There is an absence of enhancement [45].

Miscellaneous disorders

\section{Bizarre parosteal osteochondromatous proliferation} (Nora's lesion)

Bizarre parosteal osteochondromatous proliferation (BPOP), or Nora's lesion, was first described by Nora in 1983 [46]. The lesion is regarded as a reactive mass of heterotopic mineralisation arising from the periosteal aspect of an intact cortex, without medullary changes [47]. The hands and feet are most commonly involved, but location in 
Fig. 16 Hypothenar hammer syndrome. a Sagittal SE T1-WI shows a hypointense lesion superficial to the flexor tendons (arrowhead) and immediately distal to the hamulus of the hamate (asterisk). b Axial FS SE T1-WI after intravenous administration of gadolinium contrast medium shows focal aneurysmal dilatation of the ulnar artery with central thrombosis (arrow). Note only faint peripheral enhancement. c Doppler ultrasound shows intraluminal thrombosis of the aneurysm of the ulnar artery. $\mathbf{d}$ MR angiography shows multiple stenoses at the distal ulnar artery with aneurysmal dilatation with internal clot formation (arrow)
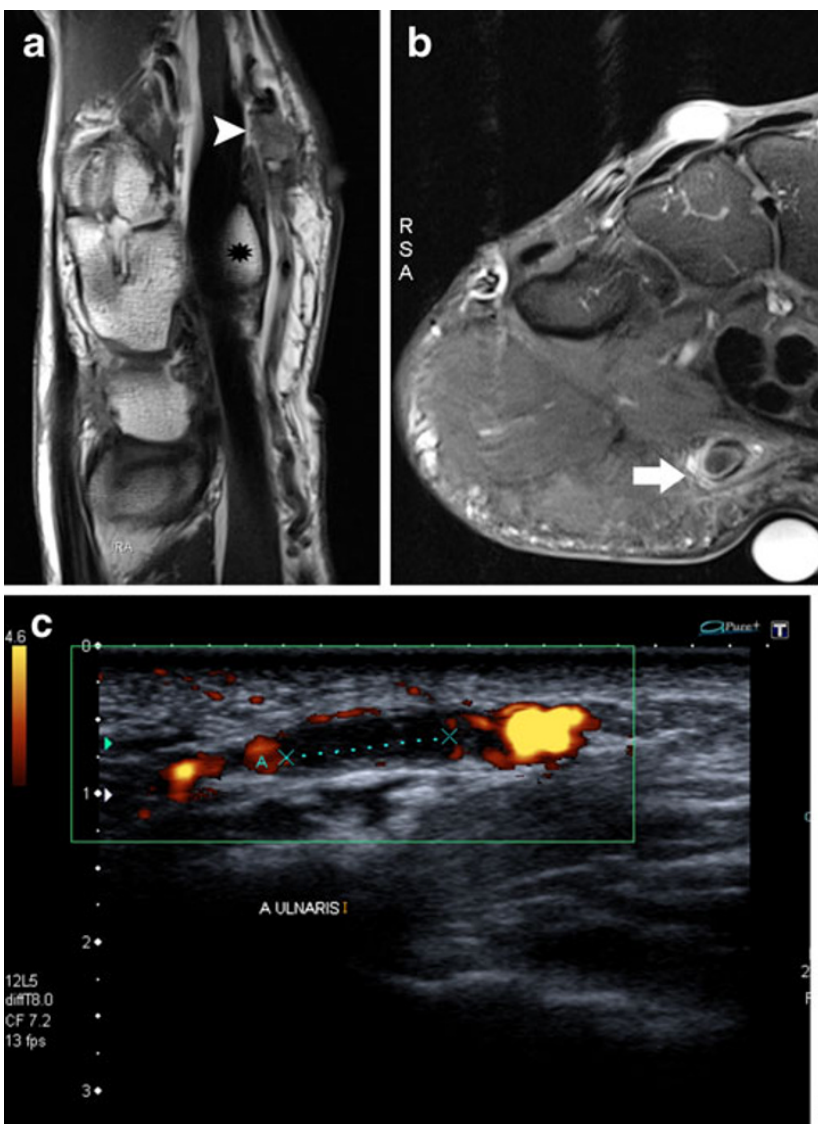
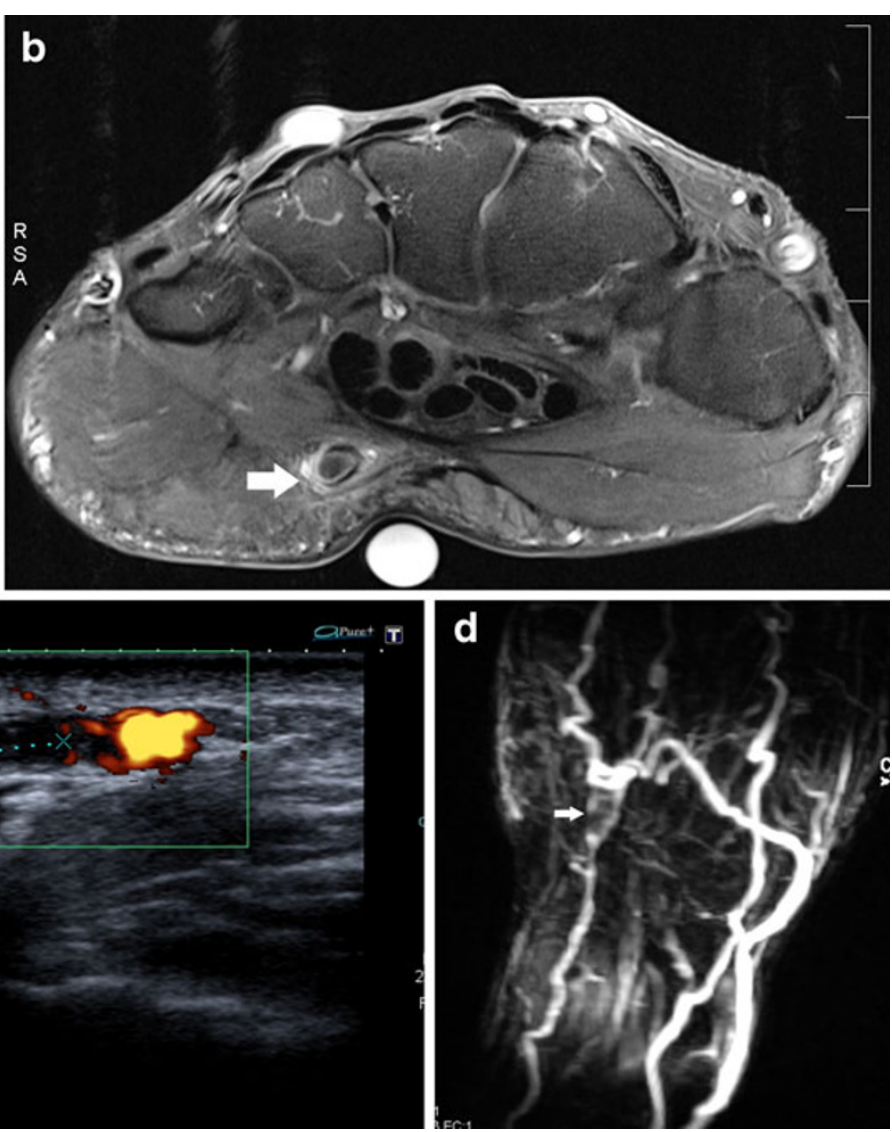

the long bones occurs in $25 \%$ of cases [47]. Ninety-two percent of the lesions of the hand are situated in the diaphysis or metaphysis of the phalanges (Fig. 18) and only $8 \%$ in the metacarpals [47]. Clinically, a soft-tissue swelling at the fingers or midhand may be present. Both pathologically and radiologically, the natural evolution of the lesion follows a spectrum of three stages (taking a maximum of 6 months) ranging from a florid reactive periostitis (stage 1) over a calcified periosteal mass lesion (stage 2) to an acquired sessile osteochondroma (stage 3 or Turret exostosis).

On MRI, the lesion has a non-specific appearance. On T1WI, the lesion shows intermediate signal intensity, whereas a variable signal is noted on T2-WI ranging from intermediate to high signal. Administration of contrast medium rarely contributes to the diagnosis. Marked contrast enhancement is the rule [47]. Recurrence after incomplete resection may occur within a period of 6 months. Because of this high recurrence rate, resection or biopsy is not recommended in early stage disease. Control examination after 6 months is the preferred management, except when the diagnosis and natural evolution are unclear [47].

\section{Focal myositis}

Focal myositis is a rare, usually self-limiting soft tissue pseudotumour [48]. It usually affects the lower extremities
$(50 \%)$, but location in the hand has occasionally been described $[49,50]$. The process is usually limited to one muscle, but one third of patients with focal myositis evolve to polymyositis [51]. Focal myositis usually presents with a painful intramuscular mass, which can grow rapidly in a few weeks. Ultrasound and MRI demonstrate focal enlargement of the muscle, with typical sparing of the internal muscle fibres $[50,52]$. T2-WI images show heterogeneous, increased signal within the affected muscle. Administration of contrast medium is of limited value because the enhancement is variable [50].

\section{Palmar fibromatosis (Dupuytren's disease)}

Palmar fibromatosis was originally described in 1831 by the French physician Dupuytren and is often referred to as Dupuytren's disease or contracture. The aetiology of palmar fibromatosis is still a matter of debate, but it is thought to represent a benign fibroblastic tumour rather than a pseudotumour. Other aetiological factors include trauma, microvascular injury, immunological processes and hereditary factors [53].

The disease is seen almost exclusively in Caucasians and is rarely seen in populations of African or Asian descent.

The disease most commonly occurs in patients over 65 years of age. men are three to four times more likely to 

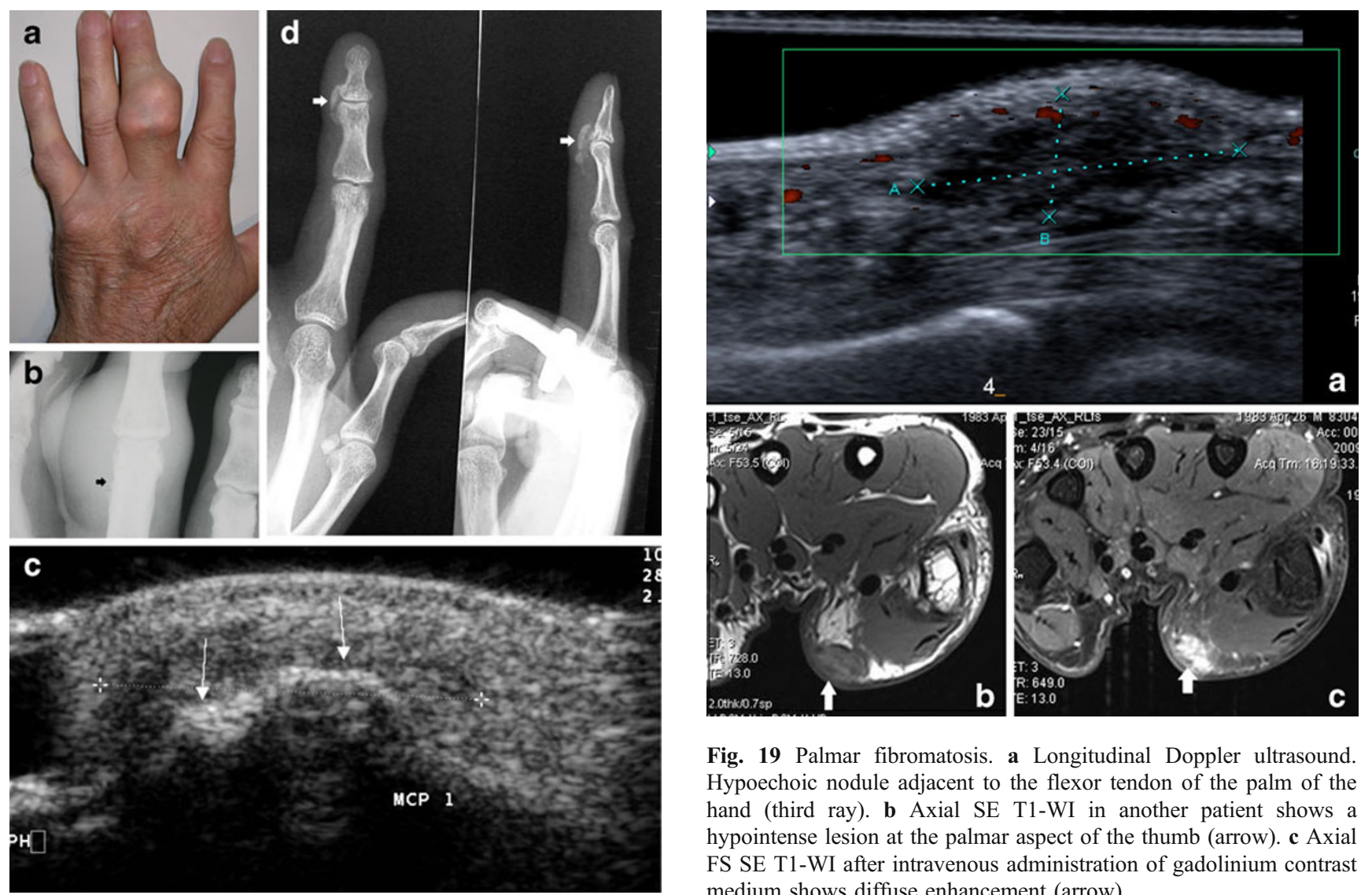

Fig. 19 Palmar fibromatosis. a Longitudinal Doppler ultrasound. Hypoechoic nodule adjacent to the flexor tendon of the palm of the hand (third ray). b Axial SE T1-WI in another patient shows a hypointense lesion at the palmar aspect of the thumb (arrow). c Axial FS SE T1-WI after intravenous administration of gadolinium contrast medium shows diffuse enhancement (arrow)

Fig. 17 Examples of pseudomasses due to metabolic diseases. a Clinical picture of tophaceous gout. Peri-articular soft-tissue swelling best seen at the proximal interphalangeal joint of the third finger. $\mathbf{b}$ Corresponding plain radiograph showing soft-tissue swelling and adjacent periosteal new bone formation (arrow). c Ultrasound of another patient with tophaceous gout at the metacarpophalangeal joint of the thumb, showing a hypoechoic mass with intralesional reflections with retro-acoustic shadowing due to monosodium urate crystal deposition (arrows). d Pseudogout (hydroxyapatite deposition disease) in another patient. Plain radiograph showing linear and amorphous calcifications at the joint capsule of the distal interphalangeal joint of the index (arrows)

be affected than women, and lesions are bilateral in 40 $60 \%$ of patients. Patients present clinically with painless subcutaneous nodules [53]. These nodules may progress slowly to fibrous cords or bands that attach to and cause traction on the underlying flexor tendons, resulting in flexion contractures of the digits. The fourth and fifth digits are most commonly involved. Patients commonly have related diseases such as plantar fibromatosis (5-20\%), Peyronie's disease, and keloid or knuckle pad fibromatosis.

Ultrasound demonstrates hypervascular, hypoechoic nodules in the palmar subcutaneous tissues, superficial to the flexor tendons (Fig. 19).

Fig. 18 Bizarre parosteal osteochondromatous proliferation (Nora's lesion). a Plain radiograph of the finger showing a turret exostosis at the dorsal aspect of the proximal phalanx of the second finger (arrowhead). b Longitudinal ultrasound shows the exostosis causing focal contour deformity of the cortical bone (arrowheads) with adjacent hypoechoic cartilage cap (callipers)
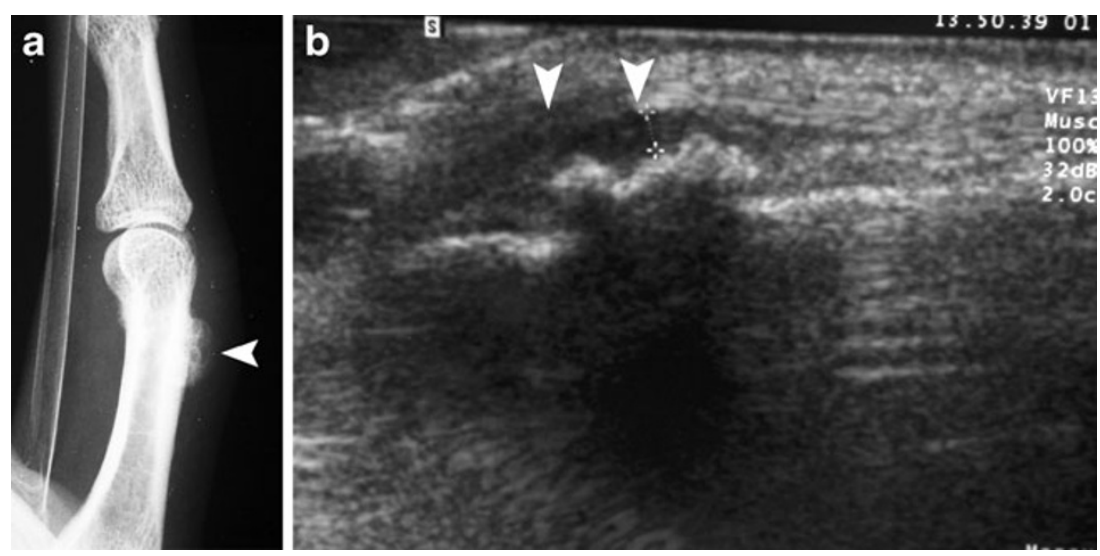
On MR imaging, there are multiple nodular or cord-like, superficial soft-tissue masses that arise from the proximal palmar aponeurosis and extend superficially parallel to the flexor tendons. Lesion length varies from 10 to $55 \mathrm{~mm}$, and lesions terminate in either a branching or nodular configuration at the level of the distal metacarpal.

The signal intensity of palmar fibromatosis is variable and related to the cellularity and collagen content of the lesion. Lesions of low signal intensity on all pulse sequences are relatively hypocellular and contain abundant dense collagen. In contradistinction, lesions of intermediate signal intensity on both T1- and T2-WI are more cellular or mixed, with less abundant collagen [53]. Lesions with a higher cellular component have been shown to have a higher local recurrence rate following local excision. After intravenous administration of gadolinium contrast medium, hypercellular lesions enhance more vividly than hypocellular lesions [53].

\section{Knuckle pads}

Knuckle pads are caused by focal fibrous thickening dorsally at the proximal interphalangeal (PIP) or metacarpophalangeal (MCP) joint, and may precede the development of palmar fibromatosis. They are usually asymptomatic and imaging features are non-specific. On MRI, nodular thickening is seen at the dorsal aspect of the PIP or MCP joint, with intermediate signal intensity compared with muscle on both pulse sequences and moderate enhancement [53].

\section{Fibro-osseous pseudotumour}

Fibro-osseous pseudotumour is a rare benign lesion that is characterised by fibroblastic proliferation with foci of osseous differentiation. The digits are frequently involved, and because of its aggressive nature, the lesion is often mistaken for a malignant lesion [54]. Imaging features are rarely reported and are non-specific.

\section{Conclusion}

Knowledge of the normal anatomy and existence and common presentation of these diseases, in combination with the relevant clinical findings (clinical history, age, location and skin changes), enables the radiologist to make the correct diagnosis in most cases, thereby limiting the need for invasive procedures. The proposed classification of these pseudotumours remains arbitrary and is still a matter of debate.

The imaging approach is often very similar to the approach to "true" soft-tissue tumoural counterparts. Biopsy should be performed only in doubtful cases.

\section{References}

1. Salgado R, De Schepper A (2008) Tumor-like Soft Tissue Lesions. In: Pope TL, Bloem HL, Beltran J, Morrison WB, Wilson DJ (eds) Imaging of the Musculoskeletal System. Saunders, Philadelphia, pp 1777-1805

2. Teh J, Whiteley G (2007) MRI of soft tissue masses of the hand and wrist. Br J Radiol 80:47-63

3. Garcia J, Bianchi S (2001) Diagnostic imaging of tumors of the hand and wrist. Eur Radiol 11:1470-1482

4. Nguyen V, Choi J, Davis KW (2004) Imaging of wrist masses. Curr Probl Diagn Radiol 33:147-160

5. Bianchi S, Della Santa D, Glauser T, Beaulieu JY, van Aaken J (2008) Sonography of masses of the wrist and hand. AJR Am J Roentgenol 191:1767-1775

6. Nahra ME, Bucchieri JS (2004) Ganglion cysts and other tumor related conditions of the hand and wrist. Hand Clin 20:249-260

7. Lowden CM, Attiah M, Garvin G, Macdermid JC, Osman S, Faber KJ (2005) The prevalence of wrist ganglia in an asymptomatic population: magnetic resonance evaluation. J Hand Surg Br 30:302-306

8. Wang G, Jacobson JA, Feng FY, Girish G, Caoili EM, Brandon C (2007) Sonography of wrist ganglion cysts: variable and noncystic appearances. J Ultrasound Med 26:1323-1328, quiz 1330-1331

9. Jebson PJ, Spencer EE Jr (2007) Flexor tendon sheath ganglions: results of surgical excision. Hand 2:94-100

10. Zamorani MP, Valle M (2007) Muscle and tendon. In: Bianchi S, Martinoli C (eds) Ultrasound of the musculoskeletal system. Springer, Berlin Heidelberg New York, pp 45-96

11. Tagliafico A, Rubino M, Autuori A, Bianchi S, Martinoli C (2007) Wrist and hand ultrasound. Semin Musculoskelet Radiol 11:95-104

12. Greendyke SD, Wilson M, Shepler TR (1992) Anterior wrist ganglia from the scaphotrapezial joint. J Hand Surg Am 17:487-490

13. Kobayashi N, Koshino T, Nakazawa A, Saito T (2001) Neuropathy of motor branch of median or ulnar nerve induced by midpalm ganglion. J Hand Surg Am 26:474-477

14. Christiaanse EC, Jager T, Vanhoenacker FM, Van Hedent E, Van Damme R (2010) Piso-hamate hiatus syndrome. JBR-BTR 93:34

15. Smith SE, Salanitri J, Lisle D (2007) Ultrasound evaluation of soft tissue masses and fluid collections. Semin Musculoskelet Radiol $11: 174-191$

16. Wong DC, Wansaicheong GK, Tsou IY (2009) Ultrasonography of the hand and wrist. Singapore Med J 50:219-225, quiz 226

17. Teefey SA, Dahiya N, Middleton WD, Gelberman RH, Boyer MI (2008) Ganglia of the hand and wrist: a sonographic analysis. AJR Am J Roentgenol 191:716-720

18. Van Zwieten KJ, Brys P, Van Rietvelde F et al (2007) Imaging of the hand, techniques and pathology: a pictorial essay. JBR-BTR 90:395-455

19. Chadha M, Agarwal A (2007) Myositis ossificans traumatica of the hand. Can J Surg 50:E21-E22

20. Ergun T, Lakadamyali H, Derincek A, Cagla Tarhan N, Ozturk A (2010) Magnetic resonance imaging in the visualization of benign tumors and tumor-like lesions of hand and wrist. Curr Probl Diagn Radiol 39:1-16

21. Tyler P, Saifuddin A (2010) The imaging of myositis ossificans. Semin Musculoskelet Radiol 14:201-216

22. Wang XL, Malghem J, Parizel PM, Gielen JL, Vanhoenacker F, De Schepper AM (2003) Pictorial essay. Myositis ossificans circumscripta. JBR-BTR 86:278-285

23. Jin W, Ryu KN, Kim GY, Kim HC, Lee JH, Park JS (2008) Sonographic findings of ruptured epidermal inclusion cysts in superficial soft tissue: emphasis on shapes, pericystic changes, and pericystic vascularity. J Ultrasound Med 27:171-176, quiz 177178 
24. Kuwano Y, Ishizaki K, Watanabe R, Nanko H (2009) Efficacy of diagnostic ultrasonography of lipomas, epidermal cysts, and ganglions. Arch Dermatol 145:761-764

25. Sconfienza LM, Silvestri E, Bartolini B, Garlaschi G, Cimmino MA Sonoelastography may help in the differential diagnosis between rheumatoid nodules and tophi. Clin Exp Rheumatol 28:144-145

26. Grant I, Mohammed P, Mahaffey PJ (2002) Granuloma annulare in the hand. J Hand Surg Br 27:556-558

27. Takeyama J, Sanada T, Watanabe M, Hatori M, Kunikata N, Aiba S (2006) Subcutaneous granuloma annulare in a child's palm: a case report. J Hand Surg Am 31:103-106

28. Chung S, Frush DP, Prose NS, Shea CR, Laor T, Bisset GS (1999) Subcutaneous granuloma annulare: MR imaging features in six children and literature review. Radiology 210:845-849

29. De Maeseneer M, Vande Walle H, Lenchik L, Machiels F, Desprechins B (1998) Subcutaneous granuloma annulare: MR imaging findings. Skeletal Radiol 27:215-217

30. Kransdorf MJ, Murphey MD, Temple HT (1998) Subcutaneous granuloma annulare: radiologic appearance. Skeletal Radiol 27:266-270

31. Letts M, Carpenter B, Soucy P, Davidson D (2000) Subcutaneous granuloma annulare of the extremities in children. Can J Surg 43:425-430

32. Shehan JM, El-Azhary RA (2005) Magnetic resonance imaging features of subcutaneous granuloma annulare. Pediatr Dermatol $22: 377-378$

33. Vandevenne JE, Colpaert CG, De Schepper AM (1998) Subcutaneous granuloma annulare: MR imaging and literature review. Eur Radiol 8:1363-1365

34. Laor T (2004) MR imaging of soft tissue tumors and tumor-like lesions. Pediatr Radiol 34:24-37

35. Navarro OM, Laffan EE, Ngan BY (2009) Pediatric soft-tissue tumors and pseudo-tumors: MR imaging features with pathologic correlation: part 1. Imaging approach, pseudotumors, vascular lesions, and adipocytic tumors. Radiographics 29:887-906

36. Blum AG, Zabel JP, Kohlmann R et al (2006) Pathologic conditions of the hypothenar eminence: evaluation with multidetector CT and MR imaging. Radiographics 26:1021-1044

37. Drape JL, Feydy A, Guerini H et al (2005) Vascular lesions of the hand. Eur J Radiol 56:331-343

38. Genchellac H, Demir MK, Unlu E, Temizoz O, Ozdemir H (2008) Hypothenar hammer syndrome: gray-scale and color Doppler sonographic appearances. J Clin Ultrasound 36:98-100
39. Winterer JT, Ghanem N, Roth M et al (2002) Diagnosis of the hypothenar hammer syndrome by high-resolution contrastenhanced MR angiography. Eur Radiol 12:2457-2462

40. Bencardino JT, Hassankhani A (2003) Calcium pyrophosphate dihydrate crystal deposition disease. Semin Musculoskelet Radiol $7: 175-185$

41. Garcia GM, McCord GC, Kumar R (2003) Hydroxyapatite crystal deposition disease. Semin Musculoskelet Radiol 7:187-193

42. Gentili A (2003) Advanced imaging of gout. Semin Musculoskelet Radiol 7:165-174

43. Yu JS, Chung C, Recht M, Dailiana T, Jurdi R (1997) MR imaging of tophaceous gout. AJR Am J Roentgenol 168:523-527

44. Ko KH, Hsu YC, Lee HS, Lee CH, Huang GS Tophaceous gout of the knee: revisiting MRI patterns in 30 patients. J Clin Rheumatol 16:209-214

45. Sheldon PJ, Forrester DM (2003) Imaging of amyloid arthropathy. Semin Musculoskelet Radiol 7:195-203

46. Nora FE, Dahlin DC, Beabout JW (1983) Bizarre parosteal osteochondromatous proliferations of the hands and feet. Am J Surg Pathol 7:245-250

47. Dhondt E, Oudenhoven L, Khan S et al (2006) Nora's lesion, a distinct radiological entity? Skeletal Radiol 35:497-502

48. Auerbach A, Fanburg-Smith JC, Wang G, Rushing EJ (2009) Focal myositis: a clinicopathologic study of 115 cases of an intramuscular mass-like reactive process. Am J Surg Pathol 33:1016-1024

49. Maguire JK Jr, Milford LW, Pitcock JA (1988) Focal myositis in the hand. J Hand Surg Am 13:140-142

50. Bashir WA, O'Donnell P The myositides: the role of imaging in diagnosis and treatment. Semin Musculoskelet Radiol 14:217-226

51. Flaisler F, Blin D, Asencio G, Lopez FM, Combe B (1993) Focal myositis: a localized form of polymyositis? J Rheumatol 20:1414 1416

52. Vanhoenacker F, Vanwambeke K, Bosmans J (2010) Pseudotumeur inflammatoire de la ceinture scapulaire. Ortho-rhumato 8:13-14

53. Murphey MD, Ruble CM, Tyszko SM, Zbojniewicz AM, Potter BK, Miettinen M (2009) From the archives of the AFIP: musculoskeletal fibromatoses: radiologic-pathologic correlation. Radiographics 29:2143-2173

54. Kaddoura I, Zaatari G (2009) Fibro-osseous pseudotumor of the thenar eminence: a rare aggressive but benign tumor. Ann Plast Surg 62:326-328 